\title{
A PHOTOSPHERIC SOLAR IRON ABUNDANCE FROM WEAK FE II LINES
}

\author{
U. Pauls ${ }^{* 1}$, N. Grevesse ${ }^{\circ}$ and M. C. E. Huber ${ }^{* 1}$ \\ * Institut für Astronomie \\ Eidgenössische Technische Hochschule \\ ETH-Zentrum \\ CH - 8092 Zürich, Switzerland \\ - Institut d'Astrophysique \\ Université de Liège \\ B - 4200 Ougrée-Liège, Belgium
}

\begin{abstract}
The high resolution and the high light-gathering power of a Fourier-transform spectrometer (FTS) afford the observation of very weak lines in laboratory spectra. Thus it became possible to determine an accurate solar iron abundance from Fe II lines that are weak in the solar spectrum: we measured the branching fractions of a few such lines whose upper levels lifetimes are known.
\end{abstract}

\section{INTRODUCTION}

The history of the solar iron abundance has been described by numerous authors (see e.g. Withbroe 1971; Grevesse 1984a,b). The solar iron abundance has repeatedly been determined over several decades. Still, uncertainties and discrepancies remain and we do not consider the problem to be solved.

To be suitable for a reliable abundance determination, Fe II lines have to be weak enough to lie on the linear part of the solar curve of growth. This requirement leads to an abundance that is independent of damping and microturbulence. But owing to the large photospheric number density of $\mathrm{Fe}^{+}$, weak Fraunhofer lines are inherently very faint and thus difficult to produce and measure in the laboratory.

\section{HIGH SIGNAL-TO-NOISE MEASUREMENTS IN THE LABORATORY}

The spectrometer ideally suited for our purposes was the solar Fourier-transform spectrometer (FTS) on Kitt Peak (cf. Brault 1985). A FTS does not scan the single spectral elements in sequence but observes the entire spectrum at once; in our case this means that (small) variations in the population of the upper levels and hence in the intensity of the emission lines do not affect the measurement of branching fractions. A second point in

1 U.P. and M.C.E.H. were guest observers at the National Solar Observatory, Kitt Peak, National Optical Astronomy Observatories, operated by the Association of Universities for Research in Astronomy, Inc., under contract with the U.S. National Science Foundation. 
favour of a FTS is its resolution; by use of path differences ranging from one to several decimeters we did always resolve the line profiles and consequently had to deal with line blends inherent in the spectrum of the light source only. Furthermore the light-gathering power of a FTS helps to perform measurements on weak lines with a laboratory light-source. Nevertheless, further steps were necessary to improve the signal-to-noise ratio (S/N) to get access to lines feeble enough for a reliable abundance determination.

As a FTS observes the whole spectrum at the same time, the noise in the recorded spectrum is proportional to the square root of the total amount of incoming photons. Consequently a drastic improvement in $\mathrm{S} / \mathrm{N}$ could be reached by use of narrow-band filters (FWHM of 1.5 and $2.0 \mathrm{~nm}$ ) - we actually achieved a factor of $10^{2}$ compared with broadband scans that extended from 0.2 to $1 \mu \mathrm{m}$. The effect of such a filter is clearly illustrated in figure 1.

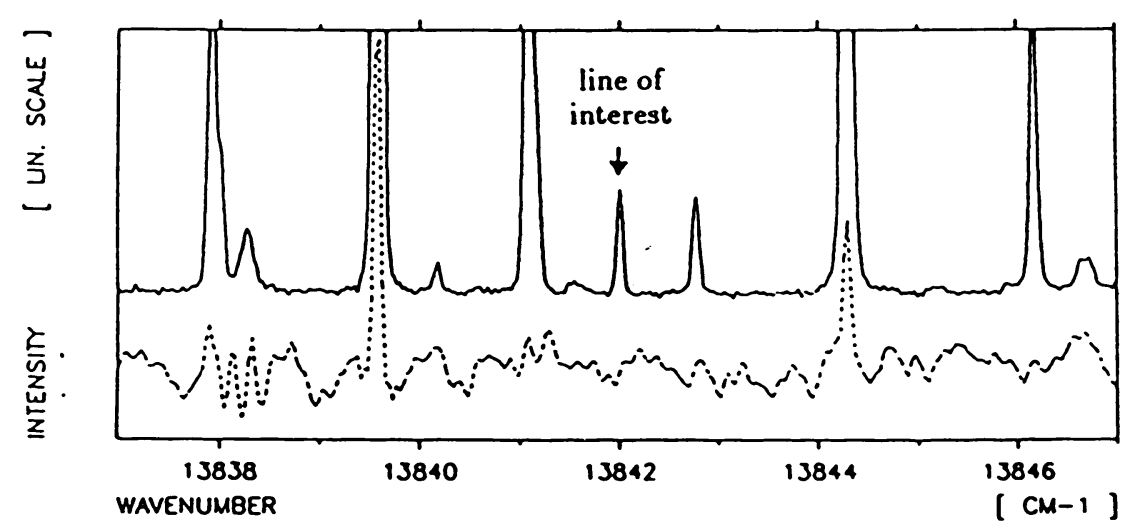

Fig. 1: Comparison of two recordings of the same spectrum by a Fourier-transform spectrometer (FTS). In one case (lower trace) the spectrum was recorded over a wide wavelength range $(0.2$ to $1 \mu \mathrm{m})$, while in the other case (upper trace) the bandwidth of the light admitted to the FTS was restricted by a narrow-band filter (full width at half maximum: FWHM $=1.5 \mathrm{~nm}$ ). The scale of the upper trace is expanded by a factor of 10 ; accordingly, the actual increase in $\mathrm{S} / \mathrm{N}$ is of the order of 100 .

The laboratory spectrum was generated in a hollow-cathode discharge run with neon as carrier gas and observed with a FTS and a 3-m grating spectrometer.

On the FTS we recorded spectra favouring the weak lines (at source conditions of 10 mbar neon and $1 \mathrm{~A}$ ). The relative radiometric sensitivity of the FTS was determined with the aid of known branching ratios of argon lines that were generated in seperate runs where argon replaced (or was added to) neon as carrier gas (Adams \& Whaling 1981).

The strong Fe II lines below $300 \mathrm{~nm}$ were measured in optically thin conditions (3 mbar neon and $0.3 \mathrm{~A}$ ) on a 3-m McPherson grating spectrometer, that had been calibrated with an argon mini-arc.

We derived the transition probabilities of three suitable Fe II lines $(\lambda \lambda \mathbf{7 2 0}-750$ $\mathrm{nm}$ ) by measuring their branching fractions and converting them into Einstein A-values by means of the lifetime of their upper levels (Hannaford \& Lowe 1983). The transition probabilities are of the order of $10^{3} \mathrm{~s}^{-1}$.

Our value for the solar photospheric iron abundance is based on the model by Holweger and Müller (1974). 


\section{CONCLUSIONS}

By judicious choice of weak Fe II lines it is possible to obtain a solar photospheric iron abundance whose accuracy is essentially dependent on the transition probabilities only. These, however, are very small and correspondingly difficult to measure. A Fourier-transform spectrometer and narrow-band filters are essential to reach the required $\mathrm{S} / \mathrm{N}$ to detect and measure such lines in the laboratory.

A final value for the solar iron abundance will be released after control measurements have confirmed our preliminary results.

ACKNOWLEDGEMENT.

We thank J.W.Brault, R.Hubbard and J.Wagner for their kind and expert support during the FTS runs. Part of this research was supported by the Schweizerischer Nationalfonds.

\section{REFERENCES.}

Adams DL \& Whaling W 1981 J.Opt.Soc.Am. 71 1036-1038

Brault JW 1985 in High Resolution in Astronomy $15^{\text {th }}$ Advanced Course of the Swiss Society of Astronomy and Astrophysics, Saas-Fee (Sauverny: Obs. de Genève) pp 1-61

Grevesse N 1984a Phys. Scripta T8 49-58

Grevesse N 1984b in Frontiers of Astronomy and Astrophysics Proceedings of the $7^{\text {th }}$ European Regional Astronomy Meeting (Firenze: Italian Astron.Soc.) pp 72-81

Hannaford P \& Lowe R 1983 J.Phys.B: At.Mol.Phys. 16 L43-L46

Holweger H \& Müller EA 1974 Solar Phys. 39 19-30

Withbroe GL 1971 Nat.Bur.Stand.(U.S.)Spec.Publ. 353 127-148 